\title{
Non-puerperal uterine inversion due to huge submucosal uterine myoma: a case report
}

\section{Simeon C. Amadi*, Peter A. Awoyesuku, Basil O. A. Altraide, Chinweowa Ohaka}

\author{
Department of Obstetrics and Gynaecology, Rivers State University Teaching Hospital, Port-Harcourt, Rivers State \\ Nigeria
}

Received: 05 August 2021

Accepted: 03 September 2021

\section{*Correspondence:}

Dr. Simeon C. Amadi,

E-mail: amachijio@yahoo.com

Copyright: () the author(s), publisher and licensee Medip Academy. This is an open-access article distributed under the terms of the Creative Commons Attribution Non-Commercial License, which permits unrestricted non-commercial use, distribution, and reproduction in any medium, provided the original work is properly cited.

\begin{abstract}
Uterine inversion is a rare clinical entity with challenging diagnosis and management. Reports of successfully managed cases contribute to knowledge and aid future management of cases among practitioners. We report a case of a 46 year old para-3 with protrusion of a mass from her vagina and vaginal bleeding of 7 days duration. She was managed as a case of chronic uterine inversion. She was resuscitated and had vaginal myomectomy, Haultin's procedure and subsequently total abdominal hysterectomy due to ischaemic necrosis of parts of the uterus.
\end{abstract}

Keywords: Uterine inversion, Non-puerperal, Sumucous myoma, Port Harcourt

\section{INTRODUCTION}

Uterine inversion is a clinical condition characterized by invagination of the fundus of the uterus through the cervical os into the vaginal canal and in some cases outside the vaginal introitus. Non-puerperal uterine inversion is rare, and it is commonly associated with masses like the benign submucous uterine myomas, attached to the fundus of the uterus. ${ }^{1,2}$ The prolapsed uterine myoma is a common non-puerperal cause of uterine inversion and other causes include leiomyosarcoma, rhabdomyosarcoma, endometrial polyps, endometrial carcinoma, and uterovaginal prolapse. ${ }^{3}$ Three pathophysiological pathways have been described for uterine inversion and include: (1) sudden emptying of the uterus, which was previously distended by a tumour, (2) thinning of the uterine walls due to an intrauterine tumour, and (3) cervical dilatation. ${ }^{4,5}$ We report a case of non-puerperal uterine inversion due to submucous uterine myoma. This is a rare clinical entity and the first case report on this pathology from our Centre. This case report will add to the existing body of literature on the clinical presentation and management of this condition.

\section{CASE REPORT}

We present, a 46 year old farmer and widow, Para-3 (with 1 living child) who presented to our accident and emergency room with 7 days history of protrusion of a mass through her vagina. This complain was preceded by a fall into a roadside drainage while walking on a flooded street. She had low back pain and mild vaginal bleeding following the protrusion of the mass. She had menorrhagia for 2 years prior to the onset of this symptom. She felt dizzy and weak during her menstruation. There was no difficulty voiding, urinary incontinence, or constipation. She made several attempts at reducing the mass without success. She noticed the mass progressively descending to the vaginal introitus hence her presentation to our centre.

She had three uneventful vaginal deliveries in the past. Her last childbirth was 17 years prior to presentation. She lost the first two babies to febrile illness at the ages of 3 and 4 years respectively. She was not desirous of future fertility. She had no significant past medical and surgical history. 
On examination at presentation, she was afebrile and other vital signs were normal. She had moderate suprapubic tenderness. Other findings on abdominal examination were normal. A mass that measured $8 \times 10 \mathrm{~cm}$ was noticed at the vaginal introitus. There was mild vaginal bleeding. The mass had areas of ischaemia but was largely within the vagina. The mass was felt emerging through the dilated cervical os with a firm cervical ring around it. The uterus was not palpable separately from the mass on bimanual examination. The adnexae were free and the pouch of Douglas was empty. There was contact bleeding. The gloved examining fingers were smeared with blood.

Differential diagnosis of prolapsed endometrial/cervical polyp and chronic uterine inversion were made. She was counselled on her condition and admitted into the Gynaecological ward. Her packed cell volume was $26 \%$. Other Full blood count parameters and the serum electrolyte urea and creatinine were normal. Her urinalysis was normal. Intra-cervical swab microscopy, culture and sensitivity revealed Moderate growth of Escherichia coli sensitive to Ceftriazone and Gentamycin. She was unable to do ultrasound scan and Magnetic Resonance Imaging (MRI) due to financial constraints. She received the antibiotics and blood transfusions. She was being worked up for examination under anaesthesia and possible polypectomy and/or correction of an inverted uterus, when on the third day of admission, the entire uterus prolapsed outside the vaginal introitus with active vaginal bleeding and hypovolaemic shock. A sub-mucous uterine myoma with a broad stalk was attached to the fundus of the uterus (Figure 1). The working diagnosis was chronic uterine inversion due to submucous uterine myoma.

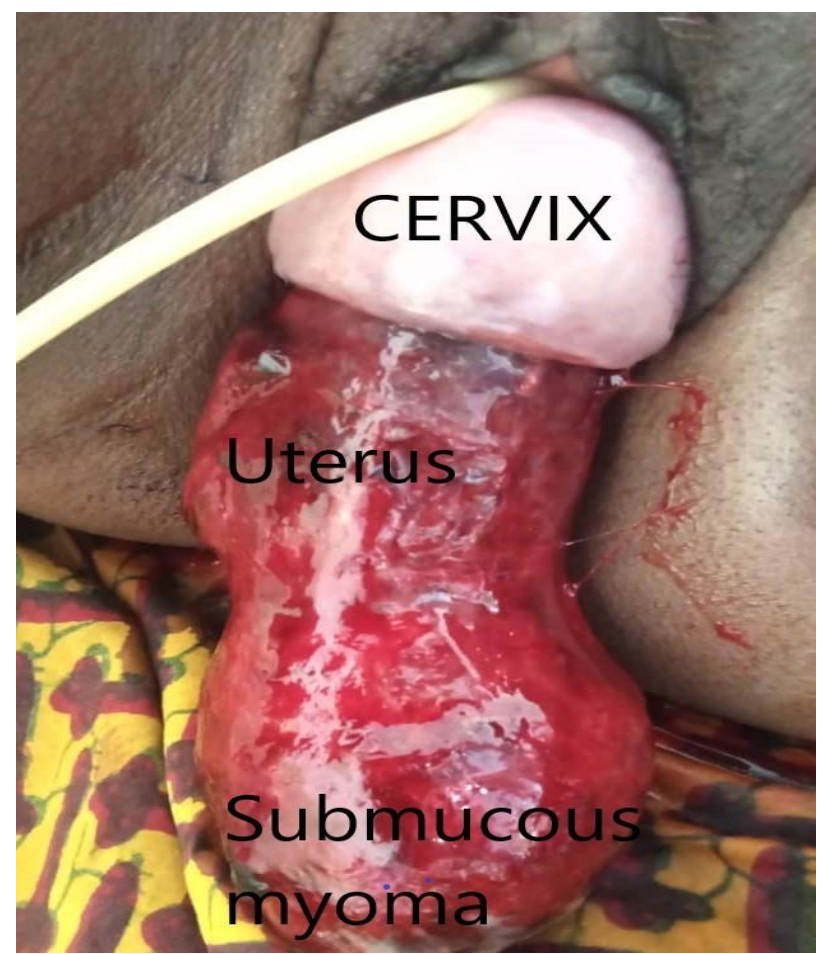

Figure 1: Prolapsed uterus.

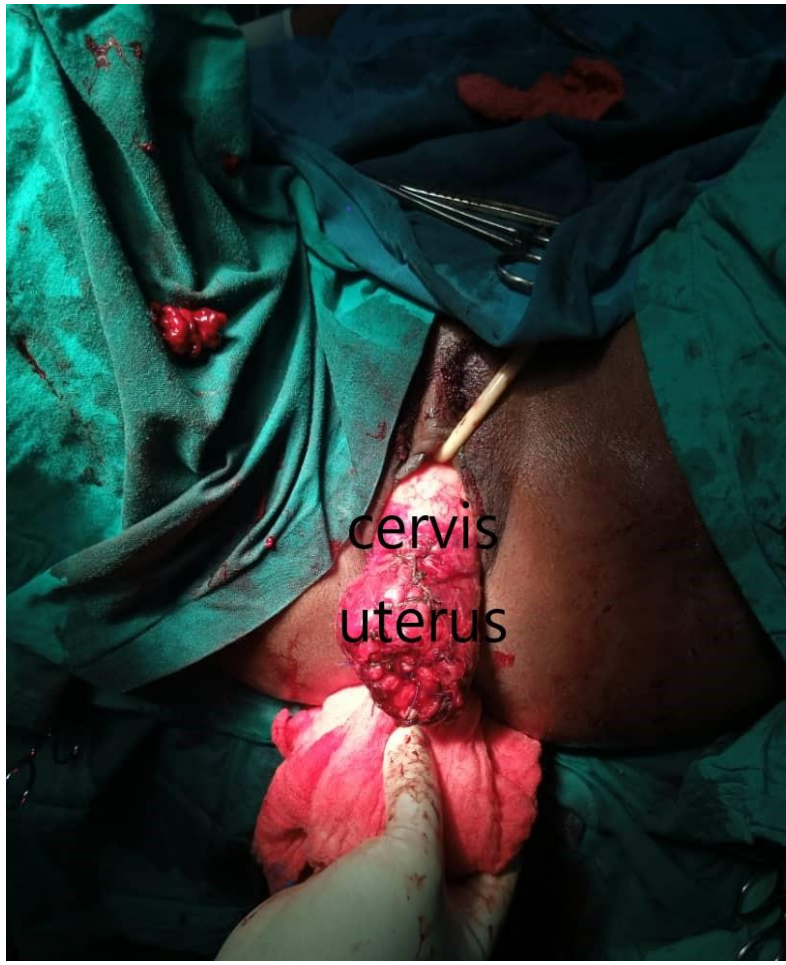

Figure 2: The prolapsed uterus after the vaginal myomectomy.

She was counselled and informed consent obtained for examination under anaesthesia and in theatre. She subsequently had vaginal myomectomy and haemostasis was secured (Figure 2). Attempts at correcting the uterine inversion after the vaginal myomectomy were unsuccessful. Due to unstable vital signs, abdominal approach for correction of the uterine inversion could not be undertaken in this theatre session. She was taken back to the ward. She was optimised with further blood transfusion and broad-spectrum antibiotics. The uterus was reduced into the vagina and packed with saline-soaked abdominal mop which was changed every 12 hours. Histology of the excised massed revealed necrotic uterine myoma. Five days later, she was taken back to the theatre and attempts at reducing the inverted uterus vaginally were unsuccessful. We resorted to exploratory laparotomy. Findings at surgery were a crater on the fundal aspect of the uterus containing the round ligaments and the fallopian tubes (Figure 3). The round ligaments and the areas of the fundus of the uterus were friable. The fallopian tubes were oedematous but the ovaries were normal. Huntington procedure was not successful. Haultain's procedure (making an incision in the posterior surface of the uterus to bisect the constriction ring in the myometrium) was undertaken to correct the uterine inversion. A total abdominal hysterectomy was eventually done for her as the uterus had areas of ischaemic necrosis. She had uneventful post-operative period. She received her broadspectrum antibiotics regularly. A unit of fresh whole blood was transfused in the immediate post-operative period. Her post-transfusion packed cell volume was $31 \%$. She was discharged home in stable clinical state on the 7 th post- 
operative day. Histology report confirmed a necrotic uterine endometrium and a necrotic uterine myoma. Two follow-up visits revealed a stable and happy woman.

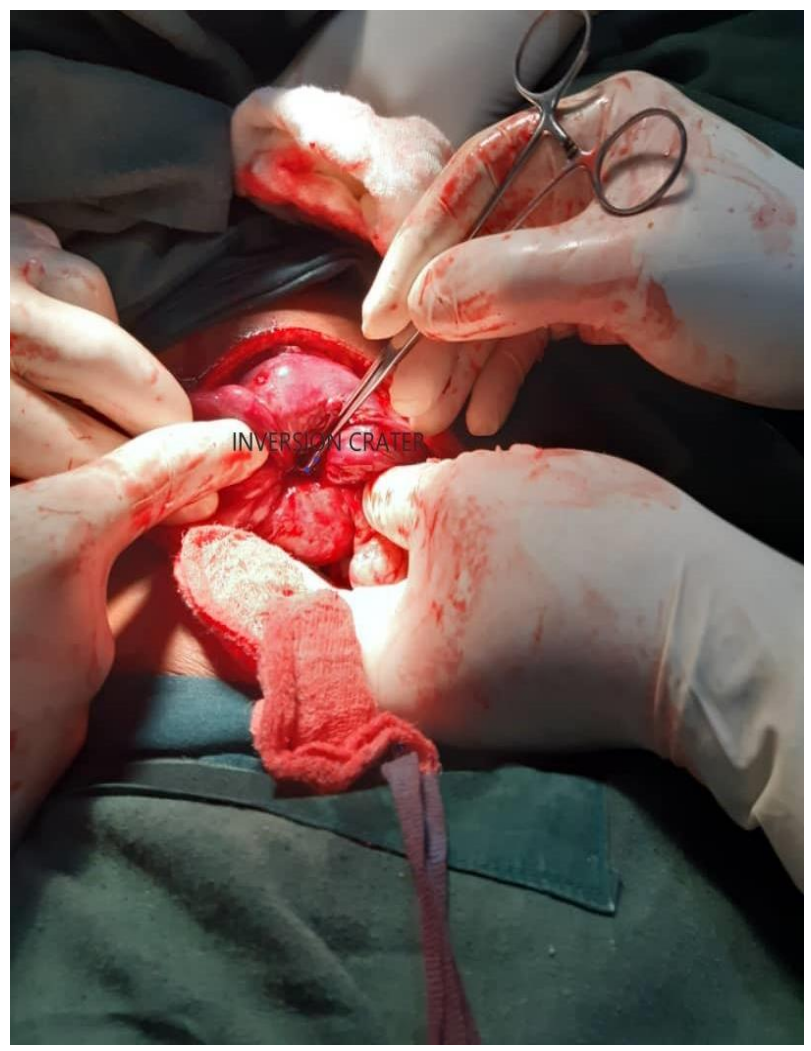

Figure 3: The crater of the uterine inversion at laparotomy; note ischaemic and friable areas of the uterine fundus.

\section{DISCUSSION}

Non-puerperal inversion of the uterus is a rare gynaecological condition and accounts for about one-sixth of all uterine inversions. ${ }^{5}$ Inversion of the uterus has been grouped into puerperal or obstetric and non-puerperal or gynaecological uterine inversions. ${ }^{6}$ Most cases of uterine inversion are puerperal inversions, whereas non-puerperal inversions are rare. ${ }^{1}$ Uterine inversion has also been categorized as acute or chronic and sub-classified as incomplete (fundus protrudes into the uterine cavity but not through the external cervical os), complete (fundus protrudes through the external cervical os), or total uterine inversion with inversion of the vagina. ${ }^{6}$ Our patient had a non-puerperal, chronic, and total uterine inversion. Nonpuerperal uterine inversions occur commonly in women aged 30 years and above. ${ }^{6}$ The index patient was 46 years. The commonest cause is submucous uterine myoma as was the case in our patient. ${ }^{3}$

Although the diagnosis can be difficult, uterine inversion should be suspected if on gynaecological examination, a mass is felt protruding through the cervix into or outside the vagina and the uterine fundus cannot be felt on bimanual examination 1.6 Acute cases are mostly symptomatic while the chronic cases are largely asymptomatic or may present with pelvic pain with a sensation of heaviness or vaginal bleeding as was seen in our patient. ${ }^{7,8}$ For the imaging techniques for diagnosis of uterine inversion, an indentation with a longitudinal hypoechoic groove of the fundal uterus under transabdominal ultrasound on a longitudinal scan is a suspicious finding of inversion. ${ }^{9}$ Magnetic resonance imaging (MRI) is not only useful in the diagnosis of uterine inversion but also helps to delineate the lesion from the nearby structures. ${ }^{10}$ Lewin et al. reported that a Ushaped uterine cavity and a thickened and inverted fundus on a T2-weighted image in a sagittal view are indicative of uterine inversion. ${ }^{11}$ These imaging investigations were not done for this patient due to financial constraints.

Most cases of uterine inversion are not always straight forward. Sometimes it is challenging to diagnose the case timely. When the case is diagnosed also, there can be a challenge with the management as it may be operated with multiple approaches, like abdominal and vaginal approaches as seen in this patient. Several surgical techniques have been described. Two abdominal (Huntington and Haultain) and two vaginal (Spinelli and Kustner) techniques have long been used to correct uterine inversion. ${ }^{12}$ Recently, laparoscopic and robotic surgeries are emerging as novel options. ${ }^{13,14}$ In the index case, a combined vaginal and abdominal approach were used. The prolapsed fundal mass was removed vaginally. This made it possible for correction of the uterine inversion abdominally using the Haultain's technique assisted with a gentle and sustained push through the vagina. Once the uterine inversion was corrected, a total abdominal hysterectomy plus bilateral salpingo-oophorectomy was done using standard procedural techniques. Hysterectomy would be technically easier on a normally positioned uterus as repositioning would restore normal anatomy that most surgeons are familiar with. ${ }^{15}$

\section{CONCLUSION}

Non-pueperal uterine inversion is a rare clinical entity. High index of suspicion, prompt and appropriate treatment are key to a successful management

\section{ACKNOWLEDGMENTS}

We acknowledge the nurses, the medical laboratory scientists, the theatre staff and all those who were involved in the successful management of this case.

\section{Funding: No funding sources \\ Conflict of interest: None declared \\ Ethical approval: Not required}

\section{REFERENCES}

1. Lupovitch A, England ER, Chen R. Non-puerperal uterine inversion in association with uterine sarcoma: 
case report in a 26-year-old and review of the literature. Gynaecol Oncol. 2005;97(3):938-41.

2. Occhionero M, Restaino G, Ciuffreda M, Carbone A, Sallustio G, Ferrandina G. Uterine inversion in association with uterine sarcoma: a case report with MRI findings and review of the literature. Gynecol Obstet Invest. 2012;73(3):260-4.

3. Katdare P, Valecha SM, Gandhewar M, Dhingra D. Chronic non-puerperal uterine inversion: recommendations for diagnosis and management. Global J Med Res. 2013;13:44-7.

4. Lascarides E, Cohen M. Surgical management of nonpuerperal inversion of the uterus. Obstet Gynecol. 1968;32:376-81.

5. Kumari A, Vidhyarthi A, Salini KM. Chronic Uterine Inversion Secondary to Submucous Fibroid: a Rare Case Report. Int J Sci Stud. 2016;3:302-4.

6. Gomez-Lobo V, Burch W. Nonpuerperal uterine inversion associated with an immature teratoma of the uterus in an adolescent. Obstet Gynecol. 2008;112:708-9.

7. Jones HW. Non-puerperal inversion of uterus. Am J Surg. 1951;81:492-5.

8. Bista KD, Rana A, Gurung G, Pradhan N, Amatya A. Towards uterine inversion: Illustration of a gradual process through three cases of submucous myoma. $\mathrm{N}$ J Obstet Gynaecol. 2006;1(2):51-4.

9. Leconte I, Thierry C, Bongiorno A, Luyckx M, Fellah L. Non-puerperal uterine inversion. J Belgian Society Radiol. 2016;100:1-5.
10. Hsieh TT, Lee JD. Sonographic findings in acute puerperal uterine inversion. J Clin Ultrasound. 1991;19:306e9.

11. Auber M, Darwish B, Lefebure A, Ness J, Roman H. Magnetic resonance imaging of non-puerperal complete uterine. Iran J Radiol. 2015;12(4):e9878.

12. Lewin JS, Bryan PJ. MR imaging of uterine inversion. J Comput Assist Tomogr. 1989;13(2):357e9.

13. Zhang X, Sun L, Chen X, Hua K. Uterus preserving reposition of non-puerperal uterine inversion under laparoscope: a case report and literature review. Gynaecol Obstet Invest. 2015;79(3):206-9.

14. Zechmeister R.J, Levey K. A. "Successful Robotically Assisted Laparoscopic Correction of Chronic Uterine Inversion" Journal of Minimally Invasive Gynecol 2011;18(5):671-3.

15. Della Corte L, Giampaolino P, Fabozzi A, di Spiezio Sardo A, Bifulco G. An Exceptional Uterine Inversion in a Virgo-intacta Patient Affected by Submucosal Leiomyoma: Case Report and Review of the Literature. The J Obstet Gynaecol Res. 2019;45(2):466-72.

Cite this article as: Amadi SC, Awoyesuku PA, Altraide BOA, Ohaka C. Non-puerperal uterine inversion due to huge submucosal uterine myoma: a case report. Int J Reprod Contracept Obstet Gynecol 2021;10:3958-61. 